\title{
Betrachtungen über die energetischen Verhältnisse total- und partiell-atmungsdefekter (TRD, PRD) haploider Mutanten von Saccharomyces cerevisiae
}

\author{
Otto Balázs \\ Physiologisches Institut der Universität Bonn, Bonn
}

\begin{abstract}
Considerations on the energetic relations in totally or partially respiratory deficient (TRD, PRD) haploid mutants of Saccbaromyces cerevisiae. Long-term observations have shown that "totally" respiration-deficient (TRD) chromosomal mutants (po+) isolated after nitrite treatment of haploid Saccharomyces cerevisiae strains very often give origin spontaneously to "partially" respiration-deficient (PRD) chromosomal mutants $\left(\mathrm{p}^{\prime} \varrho^{+}\right)$. While cultures of vegetative $(\mathrm{P} \varrho-)$ and chromosomal $\left(\mathrm{p}^{+}\right)$TRD-mutants cannot utilize non-fermentable carbon sources such as acetate, $\mathrm{p}^{\prime} \varrho^{+}$-strains can utilize acetate, although their consumption is slow and always less than that of $\mathrm{PQ}^{+}$(wild) strains with intact respiration. $\mathrm{QO}_{2}$ and $\mathrm{QCO}_{2}$ maesurements during the stationary phase on glucose substrate permit the calculation of the oxidative (OEP) and fermentative (FEP) and thus the total (GEP) energy production. From these values a strain-specific respiratory-activity-quotient (AQ) can be calculated. The group of the PRD strains shows a "continuous" transition from the TRD mutants to the "wild" Pot strains. All $\mathrm{RD}$ mutants have a tendency to maintain their total energy production values by means of an intensified fermentation. The diminution of the OEP resulting from the RD mutation exeeds that of the GEP by approximately 60 to $70 \%$. The rate of oxygen uptake is increased by a raised $\mathrm{p}_{02}$ in $\mathrm{p}^{\prime} \mathrm{g}^{+}$mutants. This is not the case with TRD mutants. Whether or not the tendency to endogenous regeneration as shown by the frequent spontaneous occurence of the process $\mathrm{p} \varrho^{+} \rightarrow \mathrm{p}^{\prime} \varrho^{+}$can be regarded as a genetic "long-term regulation" is discussed.
\end{abstract}

\section{EINLEITUNG}

Hefezellen sind bei Unterdrückung der Atmung durch bestimmte exogene oder endogene Faktoren in der Lage, ihren Energiebedarf durch Gärung zu decken und infolgedessen lebensfähig zu bleiben. Dadurch besteht die Möglichkeit, genetisch atmungsdefekte Hefezellen näher zu untersuchen.

Ursprünglich unterschied man drei Typen atmungsdefekter Hefemutanten (EpHRUSsi 1953, PitTMan et al. 1960): (1) "Vegetative" Atmungsmutanten ("petite"Mutanten), $\mathrm{P}_{\varrho}-$, mit vegetativ vererbbaren extrachromosomalen Veränderungen.

1 Die Bezeichnung der Mutantentypen wurde von Sherman \& Slonimski (1964) ubernommen: $\mathrm{P} / \mathrm{p}$ bedeutet chromosomal, $\varrho$ t/ $\varrho-$ extrachromosomal bedingte Fähigkeiten, nicht vergärbare C-Quellen zu verwerten/nicht zu verwerten. Ergänzend wurden $\mathrm{p}^{\prime}$ und $\varrho^{\prime}$ als Bezeichnung eines partiellen Atmungsdefektes eingefuhrt (BALÁS 1966). 
(2) "Spaltungsmutanten" (Chromosomal- oder Kern-Mutanten) $\mathrm{p}^{+}$, mit rezessivchromosomalem Erbfaktor. (3) Doppelmutanten, $\mathrm{p} \varrho^{-}$, mit Atmungsdefekt beider Arten.

Diese drei Gruppen atmungsdefekter Mutanten sollen zusammenfassend als total atmungsdefekte (TRD) Mutanten bezeichnet werden, da diese lediglich eine cyanresistente Restatmung (etwa 3 bis $5 \%$ der Gesamtatmung) aufweisen und nicht mehr in der Lage sind, nicht vergärbare C-Quellen (z. B. Acetat, Laktat, Glycerin und Athanol) zu verwerten und darauf zu wachsen. Die Bezeichnung "total" erstreckt sich nicht auf die cyanresistente Restatmung.

Während $\mathrm{P}_{Q}-$-Mutanten spontan entstehen oder zum Beispiel durch Acriflavinbehandlung leicht hergestellt werden können, waren po ${ }^{+}$-Mutanten nur sehr selten zu finden (PITTMaN et al. 1960). Erst durch die mutagene Einwirkung von $\mathrm{HNO}_{2}$ ist es gelungen (BALÁzs unpubliziert), Spaltungsmutanten in großer Zahl herzustellen und sie mit Hilfe der Kreuzungs-Methode (Ephrussi 1953, PItTMan et al. 1960, Balázs 1966) zu identifizieren beziehungsweise von den vegetativen Mutanten zu trennen.

Während langjähriger Beobachtungen an so erhaltenen $\mathrm{po}^{+}-$Mutanten sind sehr häufig sogenannte „Rückmutationen“ aufgetreten. Stoffwechselphysiologische Untersuchungen dieser Stämme ergaben - wie im folgenden berichtet wird - daß sie in einer vierten Gruppe atmungsdefekter Mutanten zusammenzufassen sind: (4) Partiell atmungsdefekte chromosomale Mutanten $\mathrm{p}^{\prime} \varrho^{+},(\mathrm{PRD})$, deren Atmungsfähigkeit weit über der üblichen Restatmung von TRD-Stämmen liegt, aber geringer ist, als die Atmung der atmungsintakten $\mathrm{PQ}_{Q}^{+}-$Stämme (Wild). Diese partiell atmungsdefekten (PRD)-Stämme entstanden spontan aus $\mathrm{P}^{+}-$Mutanten.

Als PRD vegetative Mutanten, $P \varrho^{\prime}$, sind wahrscheinlich die von Avers et al. (1965) beschriebenen "respiration competent" Stämme einzuordnen. Eine "Normalisierung" von PQ-Mutanten - wie sie KRAEPELIN (1964) berichtet - wurde von uns unter den gewählten Bedingungen nie gefunden.

Die Hefestämme, die zu diesem vierten Typ von RD-Mutanten gehören, können Acetat als C-Quelle langsam verwerten und sind somit durch langsames Wachstum auf Acetatsubstrat erkennbar. Diese Eigenschaft bleibt konstant vererbbar. An dieser Stelle sollen nur die energetischen Verhältnisse haploider RD-Hefestämme betrachtet werden.

\section{MATERIAL UND METHODEN}

Sämtliche untersuchten Mutanten sind Abkömmlinge zweier haploider Saccharomyces cerevisiae-Stämme entgegengesetzten Paarungstyps. Der eine ist Paarungstyp $\alpha$, Mangelmutant (ly, ileu, leu); im folgenden werden dieser Stamm und die Abkömmlinge dieses Stammes mit A und einer Katalognummer bezeichnet. Der andere ist Paarungstyp a, prototroph (wächst auf modifiziertem WICKERHAM-Nährboden, BALÁzs 1965); im folgenden werden dieser Stamm und seine Abkömmlinge mit $C$ und einer Katalognummer bezeichnet.

Die p $\varrho^{+}$- und ein Großteil der $\mathrm{P} \varrho^{--M u t a n t e n}$ wurden durch Behandlung mit salpetriger Säure hergestellt, nach der Methode, die Kaudewrtz (1959) an Escherichia coli verwendet hat. Aus dem C-Stamm entstanden durch Nitritbehandlung auch mono- 
und polyauxotrophe Stämme mit intakter (z. B. C124, C214) beziehungsweise defekter Atmung. Bei der Zusammenstellung der Ergebnisse werden diese Stämme von den autotrophen gesondert behandelt. Einige polyauxotroph gewordene Stämme wiesen einen Bedarf an 6 bis 7 Aminosäuren auf (BALÁzs 1965). PQ-Mutanten wurden auch durch Acriflavinbehandlung erhalten (Ephrussi 1953). Die Trennung der pe+- von den $\mathrm{P} \varrho^{-}-$Mutanten wurde mit Hilfe der Querstempelmethode (BALÁzs 1966) vorgenommen.

Neben dem üblichen Hefeextrakt-Glukose-Nährboden (zur Züchtung der Stämme) wurde ein Acetatnährboden mit Phenolrotindikator verwendet, auf dem TRDMutanten nicht wachsen, der aber bei atmenden Stämmen infolge oxydativen Acetatverbrauchs immer mehr alkalisch wird, was durch Rotfärbung des Indikators angezeigt wird. Der Acetatverbrauch kann quantitativ mit Hilfe potentiometrischer Titration bestimmt werden.

Q02- und QCO2-Messungen wurden durch die direkte Methode von Warburg in einer Lösung mit $0,01 \mathrm{~m}$ Glukose-Endkonzentration und einem Phosphatpuffer von PH 4,5 vorgenommen (UMBREIT, BurRIS \& STAUfFER 1964).

Sauerstoffverbrauchsmessungen auf Acetat-Nährboden wurden mittels einer neu entwickelten manometrischen Methode durchgeführt, mit der Gasstoffwechselmessungen unter Züchtungsbedingungen auch über lange Zeitdauer durchführbar sind (BALAZS 1966).

\section{ERGEBNISSE}

In Tabelle 1 sind die qualitativen Beobachtungsergebnisse über das Verhalten der verschiedenen Stämme in Schüttelkulturen auf Hefeextrakt-Glukose- und AcetatNährlösungen zusammengestellt.

Die Restglukose wurde nach 24 Stunden photometrisch bestimmt. Der Acetatver-

Tabelle 1

Glukoseverbrauch und Acetatverwertung von $\mathrm{P}^{+}+-$und RD-Stämme in Schüttelkulturen

\begin{tabular}{|c|c|c|c|c|}
\hline $\begin{array}{l}\text { Muta- } \\
\text { tions- } \\
\text { typ }\end{array}$ & $\begin{array}{l}\text { Stamm- } \\
\text { bezeich- } \\
\text { nung }\end{array}$ & $\begin{array}{c}\text { Zahl der } \\
\text { untersuch- } \\
\text { ten Stämme }\end{array}$ & $\begin{array}{l}\text { Rest- } \\
\text { glukose } \\
\mathrm{mg} / \mathrm{ml}\end{array}$ & $\begin{array}{c}\text { Zeitdauer der } \\
\text { Verfärbung des } \\
\text { Acetatnährbodens }\end{array}$ \\
\hline $\mathrm{P} Q^{+}$ & $\mathrm{C}$ & $\begin{array}{r}12 \\
6\end{array}$ & $\begin{array}{l}\sim 0,3 \\
\sim 0,5\end{array}$ & $\begin{aligned} 9 \pm 3 \mathrm{~h} \\
11 \pm 1 \mathrm{~h}\end{aligned}$ \\
\hline $\mathrm{p} \varrho^{+}$ & $\mathrm{C}$ & $\begin{array}{r}15 \\
8\end{array}$ & $\begin{array}{l}0,1 \\
0,1\end{array}$ & \multirow{2}{*}{$\begin{array}{l}\text { Keine Veränderung } \\
\text { während 10tägiger } \\
\text { Beobachtungszeit }\end{array}$} \\
\hline $\mathrm{PQ}-$ & $\mathrm{C}$ & $\begin{array}{l}17 \\
22\end{array}$ & $\begin{array}{l}0,1 \\
0,1\end{array}$ & \\
\hline $\mathrm{p}^{\prime} Q^{+}$ & $\begin{array}{l}\mathrm{C} \\
\mathrm{A}\end{array}$ & $\begin{array}{r}28 \\
7\end{array}$ & $\begin{array}{l}\sim 0,2 \\
\sim 0,2\end{array}$ & $\begin{array}{l}\text { etwa } 1 \text { bis } 4 \text { Tage je } \\
\text { nach Stamm verschieden }\end{array}$ \\
\hline
\end{tabular}


brauch dagegen wurde nur qualitativ durch die Verfärbung des zugesetzten Phenolrot-indikators (Umschlag von Gelb in Tiefrot) angezeigt und die dazu benötigte Zeitdauer angegeben. Alle Mutanten verbrauchten die angebotene Glukosemenge innerhalb von 24 Stunden fast vollständig. Signifikant unterschiedliches Verhalten zeigen sie jedoch auf Acetat-Nährboden.

Als TRD-Mutanten wurden diejenigen Stämme betrachtet, die auf Acetat-Nährboden während 10tägiger Beobachtungen keine Farbveränderungen des Indikators hervorriefen. Atmungsintakte $\mathrm{P}_{0}{ }^{\dagger}-\mathrm{Stämme}$ benötigten hingegen für eine intensive Rotfärbung maximal 12 Stunden. Stämme, die erst nach 24 Stunden Farbveränderungen bewirkten, wurden als PRD-Mutanten betrachtet.
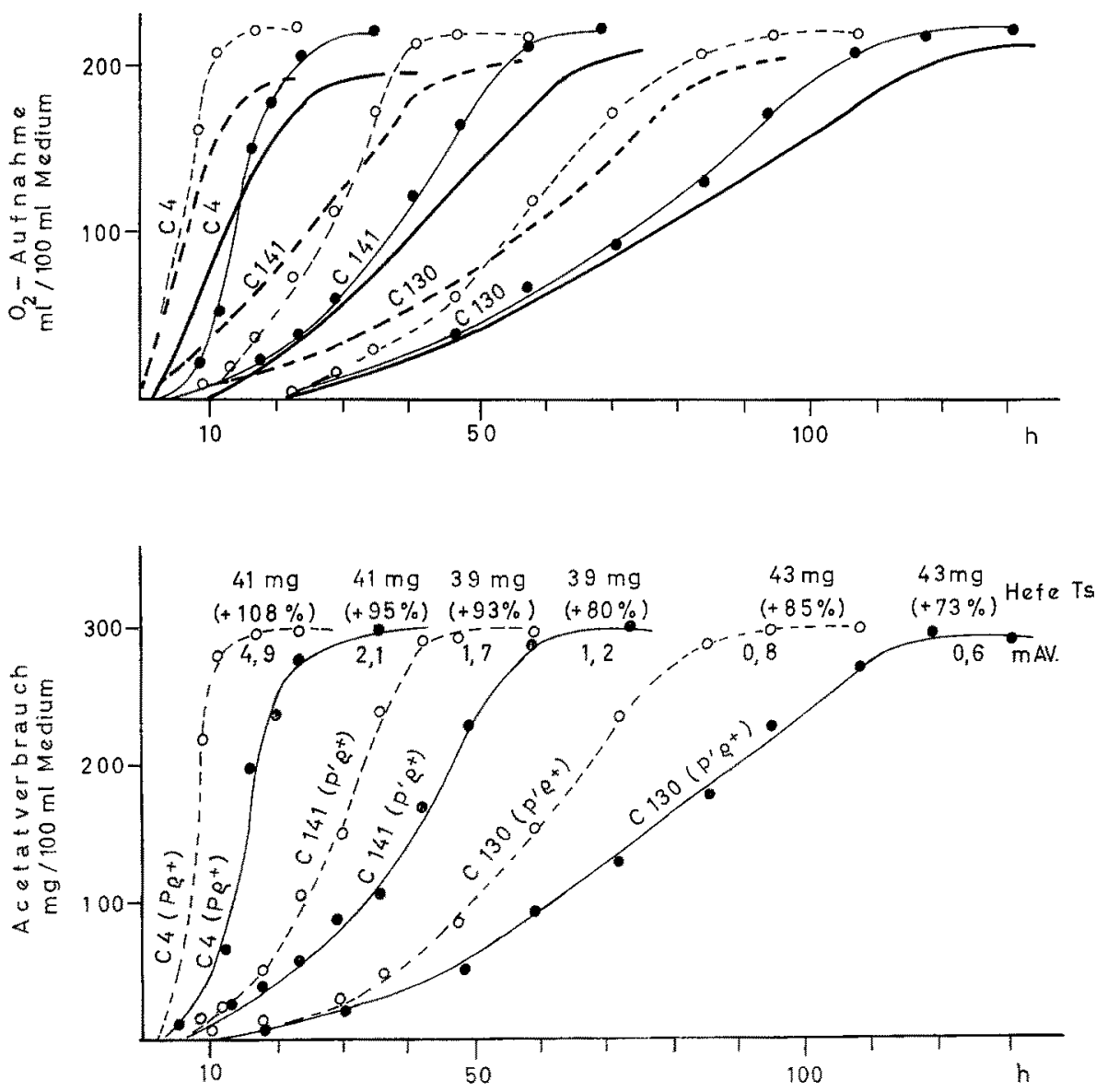

Abb. 1: Acetat- und $\mathrm{O}_{2}$-Verbrauch von $\mathrm{P}^{+}-$(C 4) und $\mathrm{p}^{\prime} \varrho^{+\ldots}$ (C 141, C 130) Stämme. Stark ausgezogene Linien: gemessen in Luft; stark ausgezogene Strichlinien: gemessen in reinem $\mathrm{O}_{2}$; schwach ausgezogene Linien: Sollwerte des $\mathrm{O}_{2}$-Verbrauchs in Luft und in reinem $\mathrm{O}_{2}$ (Strichlinie), berechnet aus den gemessenen Acetatverbrauchswerten 


\section{Acetat- und $\mathrm{O}_{2}$-Verbrauchsbestimmungen in Acetat- Nährboden unter Züchtungsbedingungen}

Um die einzelnen Stämme hinsichtlich ihrer recht unterschiedlichen Verwertung von angebotenen C-Quellen exakt miteinander vergleichen zu können, sind quantitative Messungen erforderlich. Die rasche Glukoseverwertung sämtlicher Stämme ermöglicht es, die Glukoseverbrauchsmessungen auch in Ruhephase mit Hilfe der WarburGMethode durchzuführen; dagegen ließ die langsame Acetatverwertung der PRDStämme dieses einfache Verfahren nicht zu. Auf Grund der zu erwartenden langen Meßdauer in Acetatmedium bei Zellen, die sich in der Ruhephase befinden, ist mit einer Anderung der physiologischen Beschaffenheit des zu untersuchenden Zellmaterials zu rechnen. Außerdem ergeben sich bei Anwendung der WARBurg-Methode kaum zu umgehende meßtechnische Schwierigkeiten, die ebenfalls durch die lange Meßdauer bedingt sind.

Um diese Schwierigkeiten $\mathrm{zu}$ umgehen, wurden die vergleichenden quantitativen Messungen hinsichtlich des Acetatverbrauchs nicht in der Ruhephase durchgeführt, sondern unter Züchtungsbedingungen, das heißt auf Acetat-Nährboden in Anwesenheit von Hefeextrakt. Der Acetatverbrauch wurde potentiometrisch, der $\mathrm{O}_{2}$-Verbrauch manometrisch bestimmt (BALÁzs 1966).

Die unter diesen Bedingungen ermittelten. Acetat- und $\mathrm{O}_{2}$-Verbrauchswerte ergeben einen charakteristischen, S-förmigen Kurvenablauf (Abb. 1), der wahrscheinlich durch die Zellvermehrung bedingt wird. Deshalb wurde der "mittlere Acetatverbrauch" (mAV) angegeben: Aus der gemessenen Zeitdauer, in der die gesamte angebotene Acetatmenge verbraucht wurde (Plateau), wurde der lineare Acetatverbrauch berechnet und auf $10 \mathrm{Std}$. und $1 \mathrm{mg}$ Trodkengewicht der Einsaatmenge bezogen. Die Ergebnisse sind in Tabellen 2 bis 4 (Spalte 10) zusammengefaßt.

Die auf Acetatsubstrat ermittelten $\mathrm{O}_{2}$-Verbrauchswerte, die jedoch mit den theoretischen Sollwerten nicht übereinstimmen, was auch auf einen Zellzuwachs zurückzuführen ist, verlaufen mit den Acetatverbrauchswerten parallel. Erhöhter $\mathrm{P}_{02}$ bewirkt eine Steigerung des Acetatverbrauches und gleichzeitig eine zunehmende $\mathrm{O}_{2}$-Aufnahme. Einige charakteristische Beispiele sind in Abbildung 1 dargestellt.

\section{Atmungsmessungen auf Glukosesubstrat während der Ruhephase}

Da Glukose sowohl von $\mathrm{P}^{+}-$Stämmen als auch von den TRD- und PRD-Mutanten schnell verwertet wird, besteht die Möglichkeit, die Atmung auf Glukose während der Ruhepause mit der direkten WARBURG-Methode zu messen. Es wurden stammcharakteristische Meßwerte erhalten sowohl für alle drei Gruppen der Atmungsmutanten als auch für die $\mathrm{P}_{\varrho^{+}}-$Stämme. Die Ergebnisse sind in Tabellen 2 bis 4 angegeben.

Auf Grund der ermittelten $\mathrm{QO}_{2}$ - beziehungsweise $\mathrm{QCO}_{2}$-Werte wurden die entsprechenden Energieproduktionen (OEP und FEP) berechnet, deren Summe die Gesamtenergieproduktion (GEP) ergibt. Die Berechnung der Energieproduktion beruht 


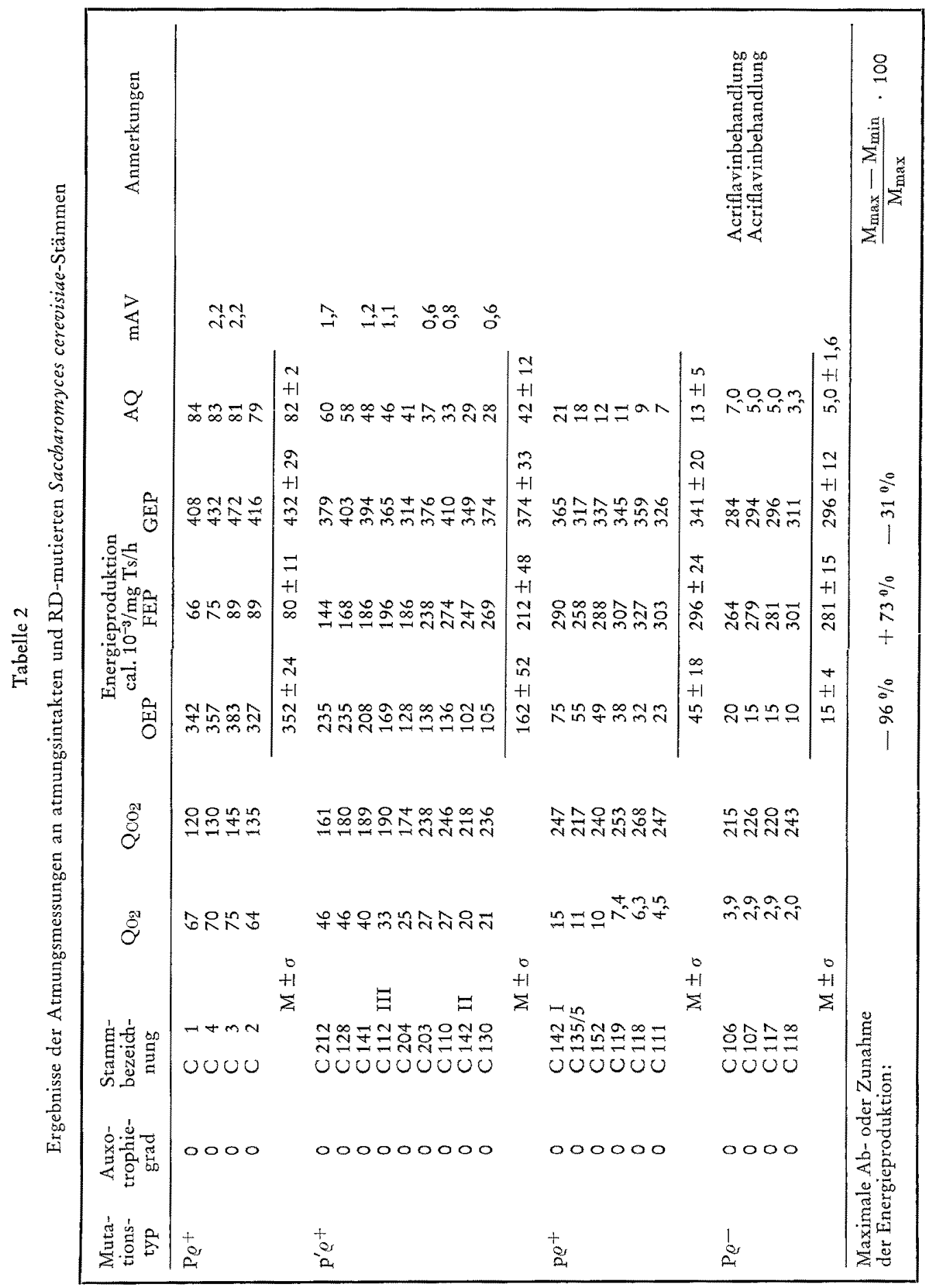




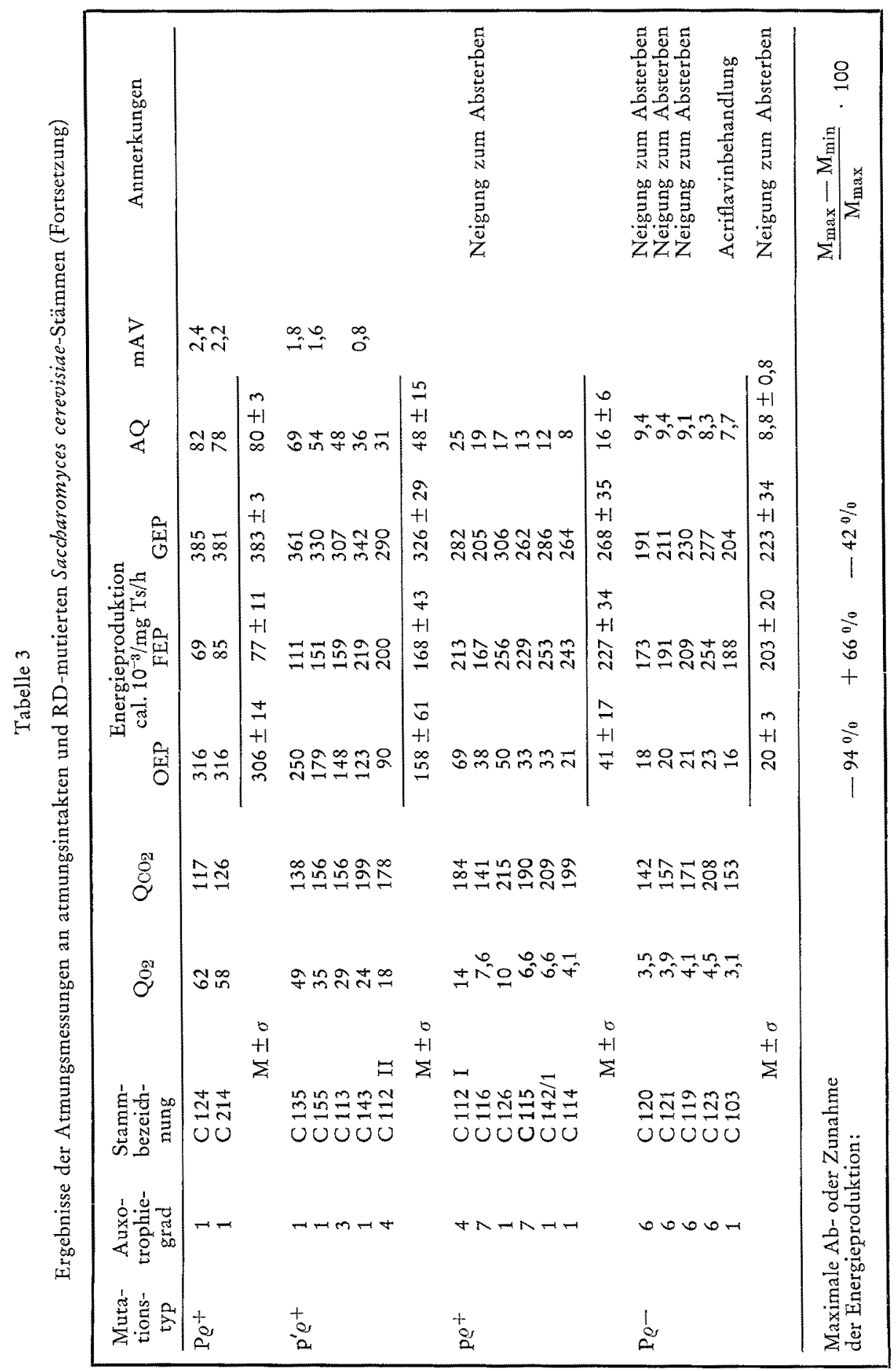




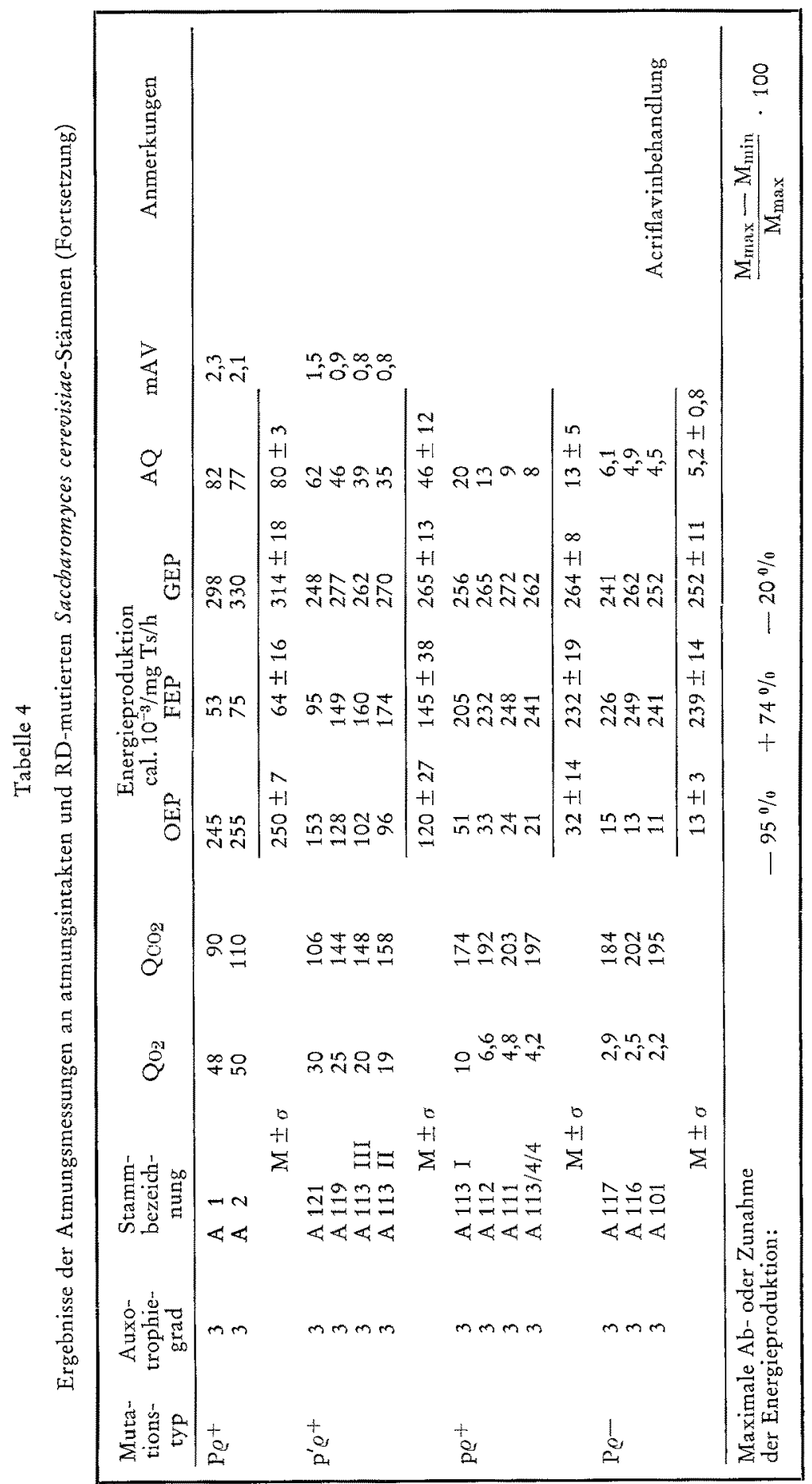




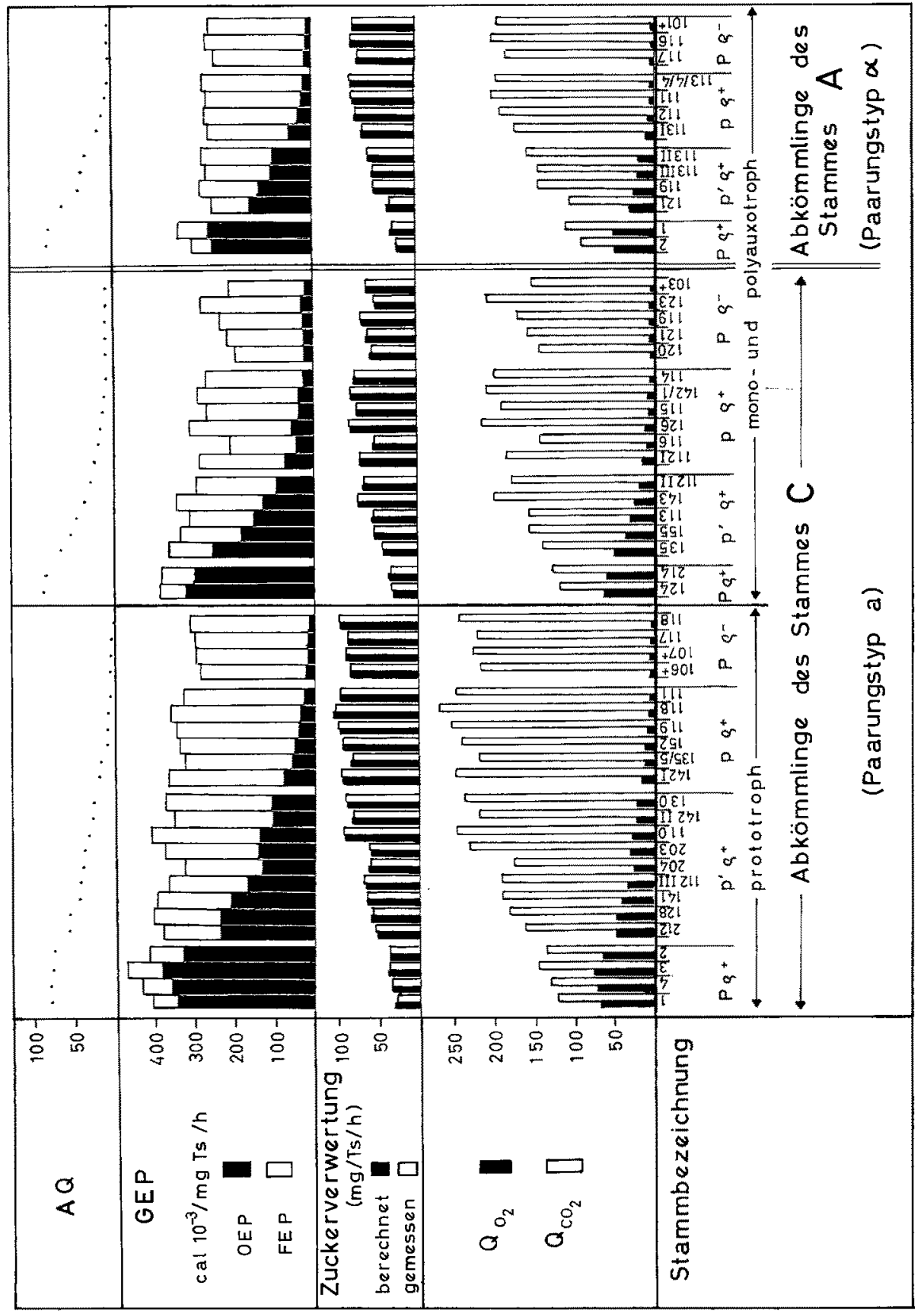

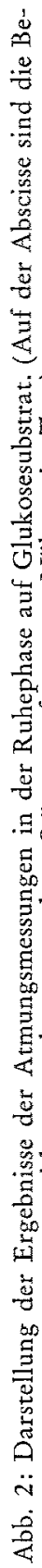


auf der Annahme, daß neben der Atmung eine rein alkoholische Gärung stattfindet. Die Richtigkeit dieser Annahme wird durch die gute Übereinstimmung der gemessenen mit den berechneten Werten für den Glukoseverbrauch (Abb. 2, mittlere Säulenreihe) bestätigt.

Berechnet man den prozentualen Anteil der durch Veratmung der Glukose produzierten Energiemenge (OEP) an der Gesamtenergie (GEP), erhält man einen charakteristischen Wert, den Atmungsaktivitätsquotienten (AQ). Dieser kann nach folgender Gleichung berechnet werden:

$$
\mathrm{AQ}=\frac{\mathrm{OEP}}{\mathrm{GEP}} \cdot 100=\frac{\mathrm{QO}_{2} \cdot 5,1}{\mathrm{Q}_{02} \cdot 5,1+\left(\mathrm{QOO}_{2}-\mathrm{QO}_{2}\right) \cdot 1,25} \cdot 100
$$

\section{DISKUSSION}

Quantitative Beobachtungen bei den aus atmungsintakten $\left(\mathrm{P}^{+}\right)$Stämmen nach Nitritbehandlung isolierten $\mathrm{p}^{+}-$Mutanten führten zu der Annahme, daß aus diesen Mutanten durch spontane „Rückmutation“ ein neuer, innerhalb der RD-Mutanten abgrenzbarer Typ entsteht: Die partiell atmungsdefekten Spaltungsmutanten $\mathrm{p}^{\prime} \varrho^{+}$. Um die Richtigkeit dieser Annahme zu prüfen, wurden vergleichende stoffwechselphysiologische Untersuchungen durchgeführt.

Die Aussage der in Tabelle 1 beschriebenen qualitativen Beobachtungen hinsichtlich der verschiedenartigen Acetatverwertung einzelner Mutantentypen wurde durch quantitative Messungen des Acetatverbrauchs wesentlich präzisiert. Die ermittelten mAV-Werte (Tabellen 2 bis 4, Spalte 10) ermöglichen, außer einer genauen Trennung der PRD-Stämme sowohl von den $\mathrm{P}^{+}+$-Stämmen als auch von den TRD-Mutanten, auch eine Art Klassifizierung der PRD-Mutanten. Die mAV-Werte liegen nämlich bei den $\mathrm{P}_{\varrho}{ }^{+}$-Stämmen bei $2,2 \pm 0,1$, während bei dem PRD-Mutanten diese Werte eine Schwankungsbreite von 0,6 bis 1,7 aufweisen.

Bemerkenswert ist, daß die höchsten mAV-Werte $(1,7)$ der PRD-Mutanten noch signifikant von denen der $\mathrm{P}^{+}-$Stämme abweichen, und daß kein einziger Fall von sogenannten Rückmutanten ermittelt werden konnte, dessen mAV-Wert dem der $\mathrm{P}_{Q^{+}}$Stämme entsprach. Noch prägnanter ist die Trennung der PRD-Stämme von den beiden TRD-Stämmen, da weder bei $\mathrm{P}^{-}{ }^{-}-$noch bei $\mathrm{p}^{+}-$Mutanten ein Acetatverbrauch nachweisbar war.

Gasstoffwechselmessungen ( $\mathrm{QO}_{2}$ und $\mathrm{QCO}_{2}$ ) auf Glukose-Substrat ermöglichen einen Uberblick über die Atmungs- beziehungsweise Gärungsverhältnisse sowohl bei den $\mathrm{P} Q^{+}-$Stämmen als auch bei allen RD-Mutanten. Sie gestatten fernerhin durch die Berechnung der freiwerdenden Energiemengen die Angabe eines stammcharakteristischen Maßes für den Atmungsdefekt, welches in Form des AQ-Wertes ausgedrückt wird.

Die voneinander abweichenden Q02-, QCO2- und GEP-Werte der Atmungsintakten $\mathrm{P}^{+}{ }^{+}$-Stämme lassen eine einheitliche Betrachtung sämtlicher Stämme nur bedingt $\mathrm{zu}$; es sind gleichwertige Gruppen zu bilden.

Die Stämme können folgendermaßen gruppiert werden: (a) In „Hauptgruppen“, die jeweils gleichwertige, nicht atmungsspezifische Eigenschaften der verschiedenartigen 
Tabelle 5

Zusammenfassung der Ergebnisse (Mittel- und Streuungswerte) von Atmungsmessungen auf Glukose-Substrat, nach genetisch verschiedenen Atmungseigenschaften geordnet

\begin{tabular}{|c|c|c|c|c|c|c|c|c|c|c|}
\hline \multirow{2}{*}{$\begin{array}{c}\text { Muta- } \\
\text { tions- } \\
\text { typ }\end{array}$} & \multirow{2}{*}{$\begin{array}{c}\text { Stamm } \\
\text { ("Haupt- } \\
\text { gruppen“) }\end{array}$} & \multirow{2}{*}{$\begin{array}{l}\text { Zahl } \\
\text { d. Ver- } \\
\text { suche }^{1}\end{array}$} & \multicolumn{2}{|c|}{ OEP } & \multicolumn{2}{|c|}{ FEP } & \multicolumn{2}{|c|}{ GEP } & \multicolumn{2}{|c|}{$\mathrm{AQ}$} \\
\hline & & & $M^{2}$ & $\pm \sigma^{3}$ & $M^{2}$ & $\pm \sigma^{3}$ & $M^{2}$ & $\pm \sigma^{3}$ & $M=$ & $\pm \sigma^{3}$ \\
\hline \multirow[t]{5}{*}{$\mathrm{Pe}^{+}$} & C prototroph & $4(3)$ & 352 & 24 & 80 & 11 & 432 & 29 & 82 & 2 \\
\hline & $\mathrm{C}$ auxotroph & 2 (1) & 306 & 14 & 77 & 11 & 383 & 3 & 80 & 3 \\
\hline & A auxotroph & $2(1)$ & 250 & 7 & 64 & 16 & 314 & 18 & 80 & 3 \\
\hline & & $8(5)$ & & & & & & & & \\
\hline & $\begin{array}{l}M_{M}{ }^{4} \\
M_{\sigma}\end{array}$ & & 315 & 20 & 75 & 12 & 390 & 23 & 81 & 2,7 \\
\hline \multirow{6}{*}{$\mathrm{pe}^{+}$} & C prototroph & $6(5)$ & 45 & 18 & 296 & 24 & 341 & 20 & 13 & 5 \\
\hline & C auxotroph & $6(5)$ & 41 & 17 & 227 & 34 & 268 & 35 & 16 & 6 \\
\hline & A auxotroph & $4(3)$ & 32 & 14 & 232 & 19 & 264 & 8 & 13 & 5 \\
\hline & & $16(13)$ & & & & & & & & \\
\hline & $M_{\text {UL }} 4$ & & 40 & & 254 & & 294 & & 13,9 & \\
\hline & $\mathrm{Mo}^{5}$ & & & 17 & & 27 & & 25 & & 5,5 \\
\hline \multirow[t]{6}{*}{$\mathrm{P} \varrho^{-}$} & C prototroph & $4(3)$ & 15 & 4 & 281 & 15 & 296 & 12 & 5,0 & 1,6 \\
\hline & C auxotroph & $5(4)$ & 20 & 3 & 203 & 29 & 223 & 34 & 8,8 & 0,8 \\
\hline & A auxotroph & $3(2)$ & 13 & 3 & 239 & 14 & 252 & 11 & 5,2 & 0,8 \\
\hline & & $12(9)$ & & & & & & & & \\
\hline & $M_{M} 4$ & & 16,5 & 33 & 171 & 32 & 238 & 24 & 6,7 & 11 \\
\hline & & & & & & & & & & $x, x$ \\
\hline \multirow{5}{*}{$\mathrm{p}^{\prime} \varrho^{+}$} & C prototroph & $9(8)$ & 162 & 52 & 212 & 48 & 374 & 33 & 42 & 12 \\
\hline & $\begin{array}{l}\text { C auxotroph } \\
\text { A auxotroph }\end{array}$ & $\begin{array}{l}5(4) \\
4(3)\end{array}$ & $\begin{array}{l}158 \\
120\end{array}$ & $\begin{array}{l}61 \\
27\end{array}$ & $\begin{array}{l}168 \\
145\end{array}$ & $\begin{array}{l}43 \\
38\end{array}$ & $\begin{array}{l}326 \\
265\end{array}$ & $\begin{array}{l}29 \\
13\end{array}$ & $\begin{array}{l}48 \\
46\end{array}$ & $\begin{array}{l}15 \\
12\end{array}$ \\
\hline & & $18(15)$ & & & & & & & & \\
\hline & $\mathrm{M}_{\mathrm{M}}{ }^{4}$ & & 151 & & 177 & & 336 & & 44,4 & \\
\hline & $\mathrm{M}_{\sigma}{ }^{5}$ & & & 51 & & 45 & & 29 & & 13 \\
\hline \multicolumn{11}{|c|}{ F-Test $\mathrm{P} \varrho^{++}+\mathrm{p} \varrho^{+}+\mathrm{P} \varrho-36(27)$} \\
\hline & $\mathrm{M}_{\sigma}{ }^{5}$ & & & 12 & & 23 & & 24,5 & & \\
\hline & $F_{15,27}$ & & & 17,7 & & $<3,8$ & nicher. & 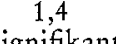 & & $<0,9$ \\
\hline & $\mathrm{p}$ & & & $<0,001$ & & $<0,01$ & nicht s & & & $<0,001$ \\
\hline \multicolumn{11}{|c|}{${ }^{1}$ In Klammern die Freiheitsgrade. } \\
\hline \multicolumn{11}{|c|}{$\sum_{i} \mathrm{x}_{\mathrm{ik}}$} \\
\hline \multicolumn{11}{|c|}{${ }^{2} M=\frac{i}{n_{i k}}$} \\
\hline \multicolumn{11}{|c|}{$\sum_{i}\left(x_{i k}-\ddot{x}_{i k}\right)^{2}$} \\
\hline \multicolumn{11}{|c|}{$\sqrt{n-1}$} \\
\hline $4 \mathrm{Mr}$ & $\sum_{k} \mathrm{n}_{\mathrm{k}} \cdot \mathrm{M}_{\mathrm{k}}$ & & & & & $\sum$ & -1 & & & \\
\hline$M_{M}$ & $=\sum_{\mathrm{k}} \mathrm{n}_{\mathrm{k}}$ & & & $M_{\sigma}$ & & $\frac{\mathrm{k}}{\sum_{\mathrm{k}}\left(\mathrm{n}_{\mathrm{k}}\right.}$ & $k-1)$ & & & \\
\hline
\end{tabular}


Stämme zusammenfassen. So entstand die Hauptgruppe der Abkömmlinge des prototrophen Stammes C, die Hauptgruppe des auxotrophen Stammes C und die Hauptgruppe der Abkömmlinge des auxotrophen Stammes A. Diese Hauptgruppen werden auf Grund der genetisch verschiedenen Atmungseigenschaften in Untergruppen unterteilt (Tab. 2, 3, 4; Abb. 2). (b) Benutzt man die verschiedenen genetisch bedingten Atmungseigenschaften als ordnendes Merkmal, werden die unter a erwähnten Hauptgruppen zu Untergruppen (Tab. 5).

Die Gesamtzahl der vorhandenen stabilen Mutanten, die also in Hauptgruppen und zusätzlich in Untergruppen geteilt werden müssen, läßt eine exakte statistische Wertung nicht zu. Die vorstehend genannten Gruppenbildungen ermöglichen aber folgende Betrachtungen: (1) Ordnet man die Stämme innerhalb der Hauptgruppen in

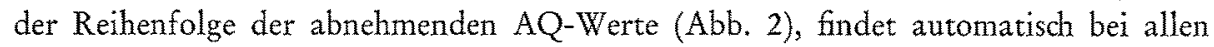
drei Hauptgruppen eine Ordnung der Stämme von den $\mathrm{P}^{+}{ }^{+}-$Stämmen über die $\mathrm{p}^{\prime} \varrho^{+}$ und $\mathrm{p} \varrho^{+}-$Stämme zu den $\mathrm{P} \varrho^{-}-$Stämmen statt. In durchschnittlich gleicher Reihenfolge nehmen auch die Qog-, OEP- und GEP-Werte ab. Dabei bilden die $\mathrm{p}^{\prime} \varrho^{+}-$Mutanten immer den Übergang von den $\mathrm{P}^{+}-$Stämmen zu den TRD-Mutanten. Gruppiert man nach den genetisch verschiedenen Atmungseigenschaften, ergibt sich folgendes (Tab. 5): Man kann innerhalb der Gruppen der Stämme $\mathrm{P}^{+}, \mathrm{P} \varrho^{+}, \mathrm{P}^{-}-$trotz unterschiedlicher "Hauptgruppenwerte“ - die Qog-, OEP- und GEP-Werte um einen Mittelwert mit relativ kleiner Streuung gruppieren. Bei den $\mathrm{P}^{\prime} \mathrm{Q}^{+}$-Mutanten dagegen ist die Schwankungsbreite signifikant wesentlich höher, als bei den anderen Stämmen (siehe F-Test, Tab. 5). Dasselbe Bild ergibt sich bei den AQ-Werten: Sie liegen für die P ${ }^{\dagger-}$ Stämme um $81 \pm 3$, für die $\mathrm{p}^{+}-$Mutanten um $14 \pm 5$, für die $\mathrm{P}^{-}-$-Mutanten um $7 \pm 1$. Die AQ-Werte der $\mathrm{p}^{\prime} \varrho^{+}-$Mutanten fallen $z$ wischen 25 und 70 , und bilden einen Obergang zwischen den oben angegebenen Grenzwerten. Aus beiden Betrachtungsweisen geht deutlich hervor, daß die Gruppe der PRD-Stämme in dem Intervall von den $\mathrm{PQ}^{+}$-Stämmen zu den TRD-Mutanten einen „kontinuierlichen " Ubergang bildet.

(2) Betrachtet man die Energieproduktion sämtlicher Stämme, erhält man folgendes Gesamtbild: Die GEP-Werte bilden sowohl in den Haupt- als auch in den Untergruppen voneinander verschiedene mittlere Niveaus mit einer gewissen stufenweisen Abnahme von den $\mathrm{P}^{+}-\mathrm{zu}$ den $\mathrm{P} \varrho^{-}-$Stämmen. Dennoch ist bei sämtlichen $\mathrm{RD}-\mathrm{Mu}-$ tanten eine Tendenz sichtbar, ihre GEP-Werte durch Erhöhung der FEP, also durch intensivierte Gärung zu erhalten. Die Streuung der GEP-Werte bei den $P^{\prime} \varrho^{+}-M u t a n-$ ten ist nicht signifikant unterschiedlich von den $\mathrm{P}_{Q^{+}}, \mathrm{P}^{+}{ }^{+}$und $\mathrm{P} \varrho^{--S t a ̈ m m e n ~(T a b . ~ 5) . ~}$ Die statistische Auswertung sämtlicher Meßwerte (Abb. 3) zeigt eine signifikante $(0,1 \%)$ Korrelation der OEP-FEP-Werte $(r=-0,82)$. Die OEP-GEP-Korrelation ist signifikant $(0,1 \%)$ positiv $(\mathrm{r}=+0,75)$. Die Abnahme der GEP-Werte beträgt im Durchschnitt bei den prototrophen C-Stämmen $31 \%$, bei den auxotrophen C-Stämmen $42 \%$ und bei den A-Stämmen $20 \%$, die OEP-Werte dagegen vermindern sich bei allen 3 Hauptgruppen um jeweils $95 \%$ (Tab. 2 bis 4).

Global betrachtet ist die Verminderung der oxydativen Energieproduktion infolge der RD-Mutation um etwa 60 bis $70 \%$ größer als die der GEP. Aus dieser Beobachtung läßt sich folgern, daß die Hefezellen hinsichtlich ihrer Energieerzeugung einen Regelmechanismus besitzen, der einer umweltsunabhängigen (also endogen deter- 
minierten) PASTEuR-Regulation entspräche. Dieses kommt darin zum Ausdruck, daß bei unzureichender OEP - infolge genetisch bedingter Veränderungen der Atmungsaktivität - die Gärung (und dadurch die FEP) in dem Maße gesteigert wird, daß die ausgefallene Energiemenge möglichst weitgehend ersetzt werden kann.

Bekanntlich wird die Energieversorgung der Zelle durch exogen beeinflußbare enzymatische Fließgleichgewichts-Systeme aufrechterhalten, wobei der jeweilige Gleichgewichtszustand mittels eines Rückkoppelungsmechanismus erhalten wird (Hess 1966). Das in den vorliegenden Untersuchungsergebnissen erhaltene Gesamtbild über

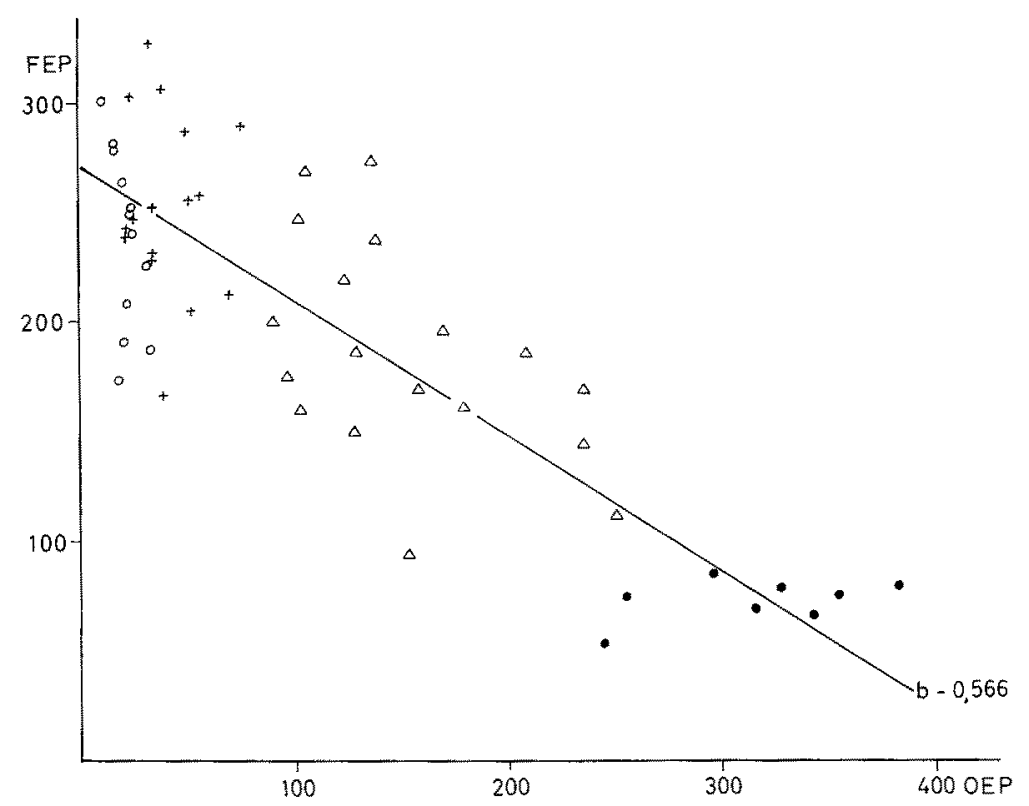

Abb. 3: Regression zwischen den OEP- und FEP-Werte sämtlicher Stämme. $0=\mathrm{P} Q^{+} ; \triangle=\mathrm{p}^{\prime} \varrho^{+} ;+=\mathrm{p} \varrho^{+} ; \bigcirc=\mathrm{P} \varrho--$ Stämme.

(Näheres im Text)

das Verhaiten atmungsdefekter Hefemutanten hinsichtlich ihrer Energieversorgung zeigt, daß dieses Fließgleichgewicht auch nach endogenen, das heißt genetisch bedingten Einwirkungen erhalten und in den meisten Fällen über einen minimalen Sollwert geregelt werden kann. Unter diesem Aspekt wäre die Energieversorgung der Zelle als genetisch determiniertes - und erblich beeinflußbares - Fließgleichgewichtssystem vorstellbar. Darin sind Atmungs- und Gärungsintensität über einen Regelmechanismus zu einer funktionellen Einheit verknüpft. Betrachtet man diesen Regelmechanismus selbst als genetisch determiniert und mutierbar, so würde das Fließgleichgewichtssystem insgesamt als Resultante von drei in Grenzen veränderlichen Größen bestimmt werden. Die bei mutagenen Einflüssen beobachtete große Zahl "letaler" Mutanten könnte somit auf ein Versagen innerhalb des komplexen Fließgleichgewichtssystems zurückgeführt 
werden, das die für die Aufrechterhaltung der Lebensfunktionen erforderliche Energie produziert beziehungsweise regelt.

(3) Der AQ-Wert gibt das Verhältnis der OEP- zu den GEP-Werten in Prozenten an. Es ist also eine von den nicht atmungsspezifischen Eigenschaften wahrscheinlich unabhängige, stammcharakteristische Größe. Mit Hilfe dieser Größe ließe sich vielleicht $z$ wischen den unterschiedlichen Saccharomyces cerevisiae-Typen ein Vergleich ziehen, und somit wären ihre verschiedenen Atmungseigenschaften einer genetischen Analyse zugänglich.

(4) Die AQ-Werte der atmungsfähigen Stämme $\left(\mathrm{P}_{\varrho^{+}}\right.$und $\left.\mathrm{p}^{\prime} \varrho^{+}\right)$weisen eine Parallelität zu den entsprechenden mAV-Werten auf (Tab. 2 bis 4). Es ist jedoch auffallend, daß bei einem AQ-Wert unter 25 Spaltungsmutanten nicht mehr in der Lage sind, Acetat zu verwerten, obwohl sie auf Glukosesubstrat noch eine erhebliche Energiemenge durch Atmung freisetzen können. Offenbar sind diese Stämme nicht mehr in der Lage, mittels ihrer Restatmung auf Acetat-Substrat die Kalorienmengen freizusetzen, die zur Aufrechterhaltung ihrer Lebensfunktionen notwendig sind. Es ergibt sich die Frage, ob etwa eine Energieschwelle vorliegen könnte. SHERman \& SlOnImski (1964) beschreiben Stämme mit der Bezeichnung $\mathrm{p}_{4} Q^{+}$, die sich auf ähnliche Weise verhalten, und bemerken, daß in diesen Mutanten alle Atmungsenzyme vorhanden sind: "They have all the cytochromes, with cytochrome $a, a_{3}$ and b present in law concentrations." Die Energieschwelle wäre also mit zu geringen (unterschwelligen) Enzymkonzentrationen zu deuten.

(5) Die Tatsache, daß auf Acetat-Substrat die Atmungsaktivität der $\mathrm{p}^{\prime} \varrho^{+-}$ Mutanten durch erhöhten $\mathrm{PO}_{2}$ in beträchtlichem Maße beeinflußt werden kann (Abb. 1), unterstützt die Annahme, daß diese Stämme sich in einer energetisch labilen Lage befinden. Thr verzögertes Wachstum auf Acetatnährboden kann also auf die von ihnen in diesem Milieu produzierbare geringe Energiemenge zurïckgeführt werden. Die Aufstellung einer brauchbaren Energiebilanz auf Acetatsubstrat und damit die Ermittlung der niedrigsten Energiemenge, die für die Erhaltung der Lebensfunktionen der Zellen in der Ruhephase notwendig ist, wird durch die Tatsache verhindert, daß die Acetatverwertung der $\mathrm{p}^{\prime} \varrho^{+}$-Stämme nur unter Züchtungsbedingungen zu bestimmen ist.

(6) Die größte Schwankungsbreite der GEP-Werte ist bei den zusätzlich Auxotrophie aufweisenden Abkömmlingen des prototrophen Stammes $C$ zu beobachten. Ein Großteil dieser Stämme wies eine 6- bis 7fache Polyauxotrophie auf (BaLÁzs 1965). Diese Polyauxotrophie ist bei einem Großteil der TRD-Mutanten allmählich nach einigen Passagen aufgetreten und blieb genetisch konstant. In diesem Zusammenhang ist zu erwähnen, daß gerade diejenigen polyauxotrophen Stämme, die auf GlukoseNährboden eine auffallend niedrige GEP zeigen, zum Absterben neigen, das heißt, sich über längere Zeit schwer oder gar nicht lebend erhalten lassen. Es wäre durchaus möglich, die von BULDER (1966) beschriebene Letalität einiger TRD-Mutanten damit zu deuten, daß eine zusätzliche Polyauxotrophie die Stätnme hindert, diejenige Energieschwelle zu erreichen, die in diesem Mangelzustand für die Aufrechterhaltung des Zellzuwachses notwendig ist.

Die stoffwechselphysiologischen Untersuchungen auf Acetat- und Glukose-Sub- 
strat beweisen, daß die durch sogenannte Rückmutation entstandenen PRD-Mutanten einen Übergang von den atmungsintakten $\mathrm{P} \varrho^{+}-$Stämmen zu den TRD-Mutanten bilden. $\mathrm{Da}$ die TRD-Mutanten im allgemeinen durch eine Veränderung in ihrem Cytochromsystem von den $\mathrm{P}^{+}{ }^{+}$-Stämmen abweichen (Ephrussi 1953, SHERMan 1963), ist mit hoher Wahrscheinlichkeit anzunehmen, daß die wohldefinierte und vererbbare neue Atmungseigenschaft der PRD-Stämme auf eine genbedingte Aktivitäts- und/oder Konzentrationsänderung der am Atmungsprozeß teilnehmenden terminalen Enzyme zurückzuführen ist (SHERMAN \& SLONIMSKI 1964). Die vergleichenden atmungsphysiologischen Untersuchungen bestätigen somit die immer mehr verbreitete Ansicht, daß der durch die ursprüngliche Mutation entstandene Defekt enzymatisch umgangen wird, und dadurch die "neuen" PRD-Stämme entstehen können.

Die hohe Zahl von aus $\mathrm{p}^{+}-$Stämmen entstandenen $\mathrm{p}^{\prime} \varrho^{+}-$Stämme (bei Strichkulturen über die gesamte Impffläche auftretend) läßt die Vermutung $z u$, daß diese Anderung nidht auf einem sekundären Mutationsvorgang („Rück“-Mutation) zurückzuführen wäre, sondern eher damit, daß - wenigstens bei einer großen Zahl der ursprünglichen $\mathrm{p} \varrho^{+}-$Mutanten - eine Art endogene Regenerationstendenz anzunehmen wäre, die der Zelle zu einer mehr ökonomischen Energieausnutzung verhilft. Dafür spricht auch der Befund, daß die Atmungsaktivität der PRD-Mutanten unterschiedlich ist und daß ihre AQ-Werte einen - wie ötters betont - sozusagen kontinuierlichen Ubbergang von den $\mathrm{P}^{+}{ }^{+}-$Stämmen zu den TRD-Mutanten bilden.

Die kybernetische Betrachtung der enzymatischen Vorgänge innerhalb der genetisch intakten Zelle brachte die Ansicht, daß die Zelle durch ein enzymatisches Rückkoppelungssystem in der Lage ist, mittels einer Art "biochemischen Schalters“ (Hess \& Brand 1965, Hess 1966) exogene schädliche Einflïsse in gewissem Rahmen innerhalb kurzer Zeit zu kompensieren. Unter diesem Aspekt könnte die Regenerationstendenz $\mathrm{p} \varrho^{+} \rightarrow \mathrm{p}^{\prime} \varrho^{+}$so interpretiert werden, daß eine Art kybernetisches Regelungssystem auch innerhalb des genetischen Informationsgehaltes der Zelle wirksam ist, wobei der OEP als informativer Sollwert für die "genetische Umschaltung" der Stoffwechselmechanismen maßgebend wäre, und daß die Zelle durch eine Art "langfristiger Regelung" in der Lage wäre, auch endogene, also genetisch bedingte Einflüsse ohne zusätzliche Mutation („Rückmutation“) zu umgehen. Der Vorgang $\mathrm{p}^{+} \rightarrow \mathrm{p}^{\prime} \varrho^{+}$wäre damit so zu deuten, daß durch den genbedingten Ausfall der Atmungsprozesse die „langfristige Regelung" in der Weise wirksam wird, daß die Zelle durch das in ihrem Informationsgehalt vorhandene Regelungssystem befähigt wird, die ausgefallenen oxydativen Prozesse enzymatisch derart zu regeln, daß durch partielle Wiederherstellung der Atmungsfähigkeit eine günstigere oxydative Energieversorgung (als Sollwert) erreicht wird.

Es ist nicht zu entscheiden, ob die auf Grund der obigen Ergebnisse angenommenen endogene "langfristige Regelung " mit einer der genetischen Arbeitshypothesen, wie multiple Allelie, Pleiotropie, oder die Wirkungsweise von Suppressor-, Repressorund Regulatorgenen in Einklang zu bringen ist. 


\section{ZUSAMMENFASSUNG}

1. Langjährige Beobachtungen zeigten, daß aus total atmungsdefekten (TRD) chromosomalen Mutanten $\left(\mathrm{po}^{+}\right)$, die nach Nitritbehandlung haploider Saccharomyces cerevisiae-Stämme isoliert wurden, sehr häufg spontan partiell atmungsdefekte (PRD) chromosomale Mutanten $\left(\mathrm{p}^{\prime} \varrho^{+}\right)$entstehen.

2. Während die vegetativen $\left(\mathrm{P} \varrho^{-}\right)$und chromosomalen ( $\left.\mathrm{P} \varrho^{+}\right)$TRD-Mutanten nicht vergärbare C-Quellen wie beispielsweise Acetat unter Züchtungsbedingungen überhaupt nicht verwerten können, zeigen die $\mathrm{p}^{\prime} \varrho^{+}-$Stämme eine Acetatverwertung, die allerdings langsam erfolgt und in allen Fällen unter dem Wert der atmungsintakten $\mathrm{P}_{\varrho}+-$ Stämme liegt.

3. Die $\mathrm{QO}_{2}$ - und $\mathrm{QCO}_{2}$-Messungen auf Glukosesubstrat in der Ruhephase ermöglichen die Ermittlung der oxydativen (OEP)- und fermentativen (FEP)- und hieraus der gesamten (GEP)-Energieproduktion. Aus diesen Werten läßt sich ein stammcharakteristischer Atmungsaktivitätsquotient (AQ) berechnen.

4. Die Gruppe der PRD-Stämme bildet einen „kontinuierlichen" Übergang von den TRD-Mutanten zu den $\mathrm{P}^{+}-$Stämmen.

5. Bei sämtlichen RD-Mutanten ist eine Tendenz sichtbar, ihre GEP-Werte durch intensivierte Gärung zu erhalten (Signifikante Korrelation zwischen OEP und FEP). Die Verminderung der OEP infolge der RD-Mutation ist um etwa 60 bis $70 \%$ größer als die der GEP.

6. Erhöhter Pog steigert bei den $\mathrm{p}^{\prime} \varrho^{+}-$Mutanten die Geschwindigkeit der $\mathrm{O}_{2}$-Aufnahme. Dies ist bei TRD-Mutanten nicht der Fall.

7. Die auf Grund des häufig spontan auftretenden Vorgangs $\mathrm{p}^{+} \rightarrow \mathrm{p}^{\prime} \varrho^{+}$angenommene endogene Regenerationstendenz wird als eine Art endogene „langfristige Regelung" gedeutet.

\section{ZITIERTE LITERATUR}

Avers, C. J., Rancourt, M. W. \& Lin, F. H., 1965. Intracellular mitochondrial diversity in various strains of Sacchayomyces cerevisiae. Proc. natn. Acad. Sci. U.S.A. 54, 527-535.

Batázs, O., 1960. Genetische Wirkung salpetriger Säure auf Saccharomyces cereviside. 1. Inaktivierende und mutagene Wirkung. (Unpubliziert)

- 1965. Auxanographisches System zur Ermittlung des Auxotrophiegrades sowie des Nährstoffbedarfes bei polyauxotrophen Mutanten yon Mikroorganismen. Arch. Mikrobiol. 50, $298-320$.

- 1966. Vergleichende Stoff wechselphysiologische Untersuchungen total- und partiell-atmungsdefekter (TRD, PRD) haploider Saccharomyces cerevisiae Mutanten. Zbl. Bakteriol. (Im Druck.)

BULDER, C. J. E. A., 1966. Lethality in respiratory deficiency and utilisation of fermentation energy in Petite negative yeasts. Arch. Mikrobiol. 53, 189-194.

Ephrussi, B., 1953. Nucleo-cytoplasmic relations in micro-organisms. Oxford Univ, pr. London, $150 \mathrm{pp}$.

Hess, B., 1966. Kontrollmechanismen der Glykolyse. Helgoländer wiss, Meeresunters. 14 (im Druck).

- \& BRAnd, K., 1965. Enzyme and metabolite profiles. In: Control of energy metabolism. Ed. by B. Chance, R. W. Estabrook \& J. R. Williamson. Acad. pr., New York, 111-122. 
KAUDEWITZ, F., 1959. Inaktivierende und mutagene Wirkung salpetriger Säure auf Zellen von Escherichia coli. Z. Naturf. 14b, 528-537.

Kraepelin, G., 1964. Normalisierung des Atmungsdefektes bei Hefe. Rückführung stabilisierter RD-Mutanten in voll atmungsfähige Normalzellen. Arch. Mikrobiol. 48, 299-305.

Nagai, S., Yanagishima, N. \& NagaI, H., 1961. Advances in the study of respirationdeficient (RD) mutation in yeast and other microorganisms. Bact. Rev. 25, 404-426.

Ogur, M., Lindegren, G. \& Lindegren, C. C., 1954. A simple screening test for genetic studies of respiration deficiency in yeast. J. Bact. 68, 391-392.

Pittman, D., Webr, J. M., Roshanmanesh, A. \& Coker, L. E., 1960. Evidence for the genetic control of photoreactivation. Genetics, Princeton 45, 1023-1037.

Ravt, C., 1953. A'cytodhrome deficient mutant of Saccharomyces cerevisiae. Expl Cell Res. 4, 295-305.

Sherman, F., 1963. Respiration-deficient mutants of yeast. 1. Genetics. Genetics, Princeton 48, 375-385.

- \&. Slonimski, P. P., 1964. Respiration-deficient mutants of yeast. 2. Biochemistry. Biochim. biophys. Acta 90, 1-15.

Umbreir, W. W., Burris, R. H. \& Stauffer, J. F., 1964. Manometric techniques. 4th ed. Burgess, Minneapolis, Minn., 305 pp.

\section{Diskussion im Anschluß an den Vortrag BaLÁzs}

Hess: Können Sie uns sagen, unter welchen Sauerstoffdrucken Sie gearbeitet haben?

BALÁzS: Es wurde mit Luft und mit reinem Sauerstoff gearbeitet, also bei etwa 150 beziehungsweise 760 Torr $\mathrm{P}_{02}$.

Hess: Ich stelle die Frage, weil ich wissen will, ob die Cytochrommangelmutanten durch Sauerstoff oder durch andere Substrate wie Acetate induziert werden können. Es könnte sein, daß die Cytochromsynthese lediglich deprimiert ist.

BALÁzs: Die Versuche auf Acetat-Substrat mit erhöhtem pos könnten darauf deuten, daßs eine $\mathrm{O}_{2}$-Induktion der Cytochromsysteme bei atmungsintakten und partiell atmungsdefekten Stämmen durchaus im Bereich der Möglichkeit liegt. Daß Acetat eine induktive Wirkung ausübt, wäre unter den verwendeten Versuchsbedingungen unwahrscheinlich; man könnte eine solche Wirkung höchstens dem erhöhten $\mathrm{P}_{\mathrm{O} 2}$ zuschreiben. Wenn überhaupt eine erhöhte Acetatverwertung als Folge einer Induktion betrachtet werden kann, ist es bemerkenswert, daß die Erhöhung der Acetatverwertung und der $\mathrm{O}_{2}$-Aufnahme bei den PRD-Mutanten nur innerhalb offenbar genetisch bestimmter Grenzen stattfinden kann. Mit anderen Worten: die Möglichkeit einer Erhöhung der Atmung durch eventuelle Sauerstoff-Induktion ist je nach Stamm limitiert, woraus man schließen könnte, daß die Atmungsintensität der Stämme mit verschiedenen $\mathrm{AQ}$ Werten auf jeweils unterschiedlichen Niveaus genetisch festgelegt ist. Total atmungsdefekte Mutanten (weder $\mathrm{p}^{+}$noch $\mathrm{P} \varrho-$ ) weisen auch nach erhöhtem $\mathrm{P}_{\mathrm{O} 2}$ keine meßbare Acetatverwertung auf; man kann wohl annehmen, daß bei diesen Mutanten kein induzierbares System vorliegt. Die Anderung $\mathrm{p} \varrho^{+} \rightarrow \mathrm{p}^{\prime} \varrho^{+}$kann wohl nicht auf Induktion beruhen, da dieser Vorgang ja spontan, in Luft und auf Glukose-Substrat beobachtet worden ist. Es wäre durchaus interessant, zu untersuchen, ob diese von uns auf eine "genetische Schalterwirkung" zurückgeführte Anderung zum Beispiel durch erhöhten $\mathrm{P}_{\mathrm{O}_{2}}$ oder durch Acetat zu beeinflussen ist. 